\title{
Structure Investigation on Liquid Tellurium by X-Ray and Neutron Scattering
}

\author{
Walter Hoyer, Hartmut Neumann, and Manfred Wobst \\ Technische Universität Chemnitz, Fachbereich Physik, Chemnitz, Germany
}

Z. Naturforsch. 47a, 833-840 (1992); received May 8, 1992

\begin{abstract}
We present the results of neutron and X-ray scattering experiments on liquid tellurium. No dependence on temperature of the radius and the coordination number of the first neighbour shell has been found in the temperature range between $460^{\circ} \mathrm{C}$ and $550^{\circ} \mathrm{C}$. We investigate the influence of the truncation of the Fourier transformation on the determined coordination number of nearest neighbours and on the shape of the second coordination maximum at both, the lower and upper integration limit. The structure of liquid tellurium can be described in terms of a chain-like atomic arrangement with two bonds per atom.
\end{abstract}

Key words: Neutron scattering, X-ray scattering, Short-range order, Liquid tellurium, Termination effect.

\section{Introduction}

One of the basic problems in condensed matter physics is the explanation of the solid-liquid phase transition. The comparison of crystalline and liquid structures is one important key for the understanding of the nature of the chemical bond between atoms. While liquid metals are relatively well understood, covalently bounded substances show a broader variety of behaviours. The melting of $\mathrm{Si}$ and $\mathrm{Ge}$ is characterized by distinct changes of distance and coordination number of nearest neighbours. On the other hand, the essential structural features of the solid are preserved in the liquid state for the group VI elements $\mathrm{S}$ and Se. The same behaviour is found for arsenic. Distance and coordination number of first neighbours in liquid As are very close to the values of the crystalline phase [1]. Much attention has been devoted to liquid tellurium, which maintains a special place in research on the structure and properties because of the complex electronic and thermodynamic behaviour of numerous Te-based alloys. Central to an understanding of these properties is the detailed nature of the bonding and structure.

The purpose of this paper is to present the results of recent X-ray and neutron scattering experiments and to discuss them with respect mainly to the question of the dominance of twofold or threefold coordination

Reprint requests to Prof. Dr. W. Hoyer, Technische Universität Chemnitz, Fachbereich Physik, PSF 964, O-9010 Chemnitz, Germany. and to the nature of the maximum, shoulder or at least asymmetry in the region $3.4-3.8 \AA$ of the pair correlation function. We examine the influence of the lower and upper integration limits of the Fourier transformation on the structural characteristics of liquid Te.

\section{Experimental Details and Results}

Neutron scattering experiments have been carried out on the double-axis spectrometer of the research reactor at Rossendorf; the general experimental conditions have been given by Neumann et al [2]. The neutron wavelength was $\lambda=0.96 \AA$ and the accessible $Q$-values $(Q=(4 \pi / \lambda) \sin \theta$, where $\theta$ is the half scattering angle) range from $1.0 \AA^{-1}$ to $9.3 \AA^{-1}$. The measurements were performed by step scanning in angular increments of $10^{\prime}$. The data were converted to equidis$\operatorname{tant} Q$-values by fitting short segments of the spectra (about $2^{\circ}$ ) with a least-squares routine. The samples were contained in sealed quartz tubes (internal diameter $15 \mathrm{~mm}$, wall thickness $1 \mathrm{~mm})$. The overall uncertainty in the sample temperature $\left(480\right.$ and $\left.550^{\circ} \mathrm{C}\right)$ was smaller than $5 \mathrm{~K}$. The data correction procedure has been described in the previous papers [2,3].

The X-ray diffraction experiments were carried out with a $\theta-\theta$ diffractometer using $\mathrm{MoK}_{\alpha}$ radiation which has been selected by a focusing graphite monochromator placed in the diffracted beam. The $\mathrm{X}$-rays were scattered at the free surface of the melt. Above about $500{ }^{\circ} \mathrm{C} \mathrm{X}$-ray measurements with a free

0932-0784 / $92 / 0700-0833 \$ 01.30 / 0$. - Please order a reprint rather than making your own copy. 
liquid tellurium surface (the graphite crucible with the sample (area $25 \times 40 \mathrm{~mm}^{2}$ ) is placed in an Ar-filled high temperature chamber) are rather difficult because of the high vapour pressure of the sample. In order to prevent detectable angular dependent falsifications, caused by evaporated tellurium which contaminates the Be X-ray windows of the high temperature chamber, the scattering intensity has been measured in relatively narrow regions of momentum transfer $\left(1-8 \AA^{-1}\right.$, $\left.4-11 \AA^{-1}, 7 \AA^{-1}-Q_{\max }\right)$ by repeated runs. $Q_{\max }$ is $13.65 \AA^{-1}$ at $460{ }^{\circ} \mathrm{C}$ and is $12.05 \AA^{-1}$ at $500^{\circ} \mathrm{C}$. The sample temperatures were held constant within an error of about $1 \mathrm{~K}$. Conventional data reduction has been applied for polarization and incoherent scattering.

In Fig. 1 the structure factors from neutron scattering are shown. A marked "triangle like" first peak, as pointed out by Takeda et al. [4], has not been observed. The height of the first peak is slightly reduced at the higher temperature. Compared with the first two peaks the third and fourth peaks are rather broad. The weak splitting, visible on the third peak, may be caused by residual scattering contributions from the quartz container. The asymmetry of the fourth peak is confirmed by the X-ray result. The structure factors from the X-ray experiments are drawn in Figure 2. The differences between the results of X-ray and neutron scattering are mainly caused by the rather sensitive absorption correction of the neutron experiment.

The density values required for the Fourier transformation were taken from the data published by Thurn and Ruska [5]. The calculated pair correlation functions are shown in Figs. 3 and 4 for neutron and $\mathrm{X}$-ray scattering, respectively. The lower integration limit was $Q=0 \AA^{-1}$ in both figures. The upper integration limit was $Q=9.35 \AA^{-1}$ for the neutron data and $Q=11.75 \AA^{-1}$ for the X-ray scattering. No modification functions or repeated transformation have been employed. The general shape of the functions is very similar. The first peak is slightly broader for the neutron diffraction because of the smaller $Q_{\max }$. Drastic temperature dependent changes are not observed. Each of the functions exhibits a well pronounced shoulder on the second peak. Its position is at somewhat lower $r$-values in the X-ray functions than in the neutron functions. Because of the different upper integration limits this behaviour indicates that the corresponding $r$-region is affected by truncation errors.

The position of the first coordination shell and its coordination number are given in Table 1 . The coordi-

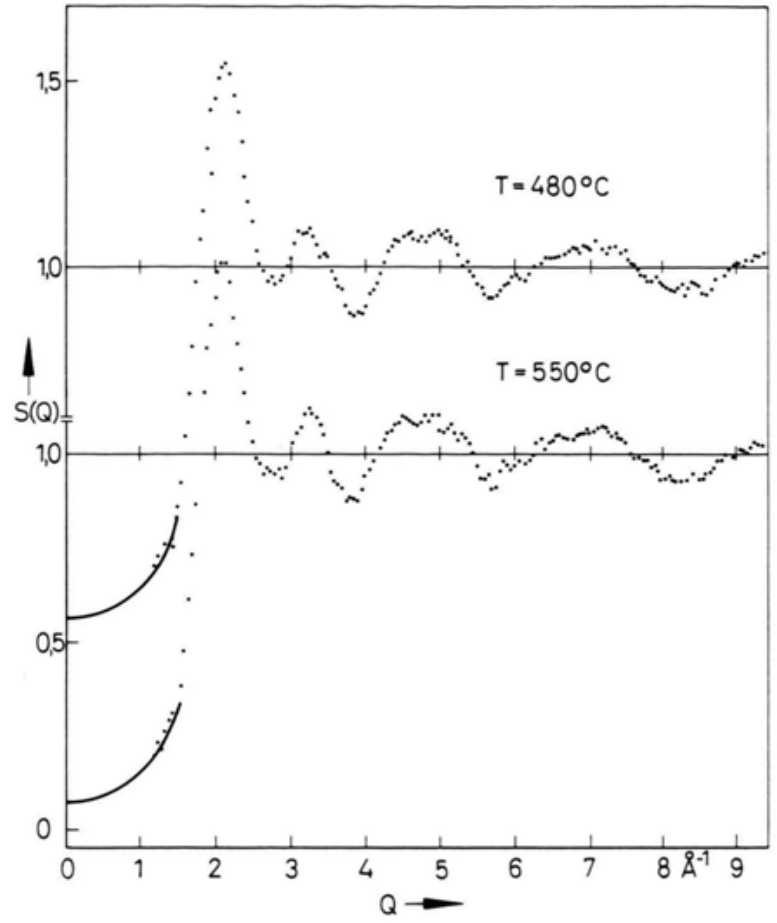

Fig. 1. Structure factors $S(Q)$ by neutron scattering. $\cdots$ experimental points, - extrapolation.

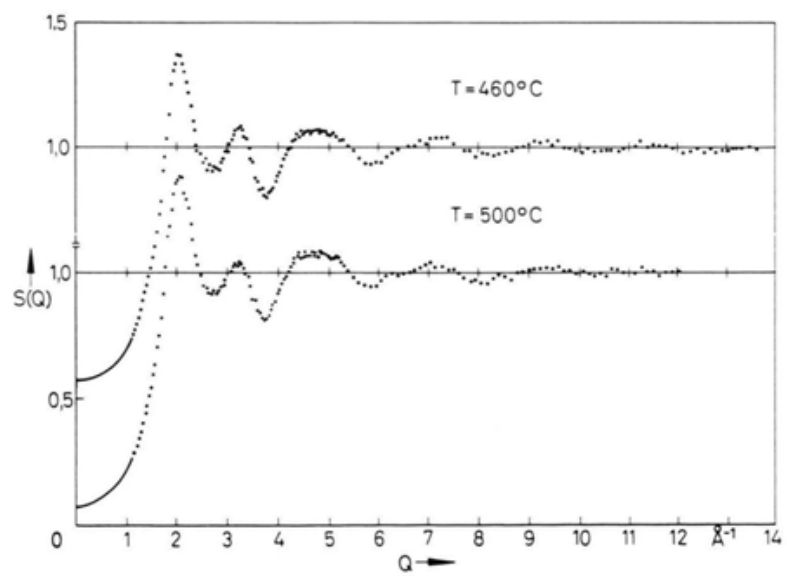

Fig. 2. Structure factors by X-ray scattering. $\cdots$ experimental points, $\longrightarrow$ extrapolation.

nation number has been determined by integration up to the first minimum of the radial distribution function. Position and coordination number show no dependence on temperature. The somewhat higher coordination numbers determined from the neutron functions are caused by the lower $Q_{\max }$-values, as will be shown in more detail below. 


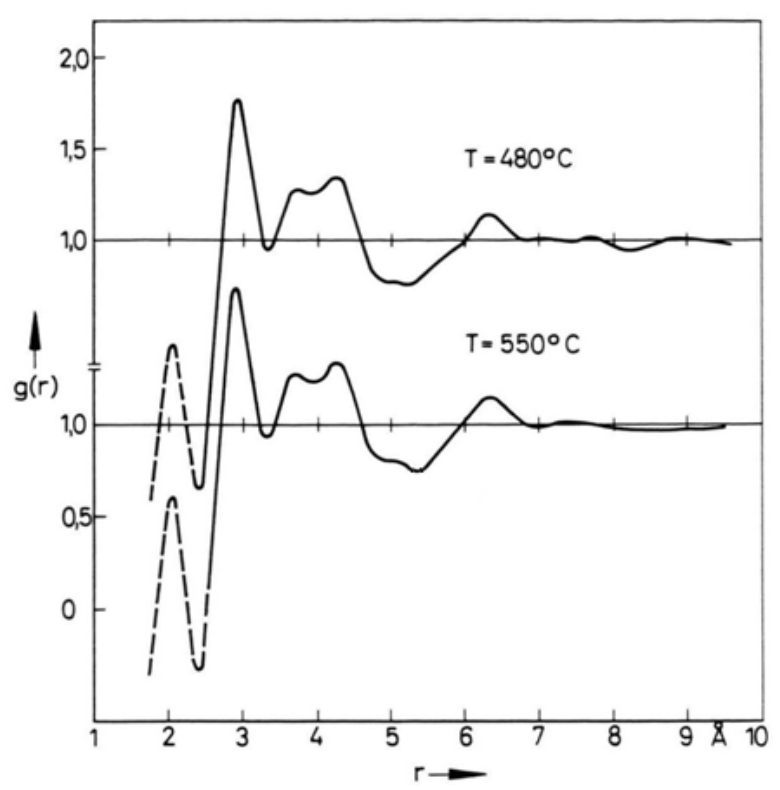

Fig. 3. Pair correlation functions calculated from the neutron scattering results shown in Fig. $1,0<Q<9.35 \AA^{-1}$.

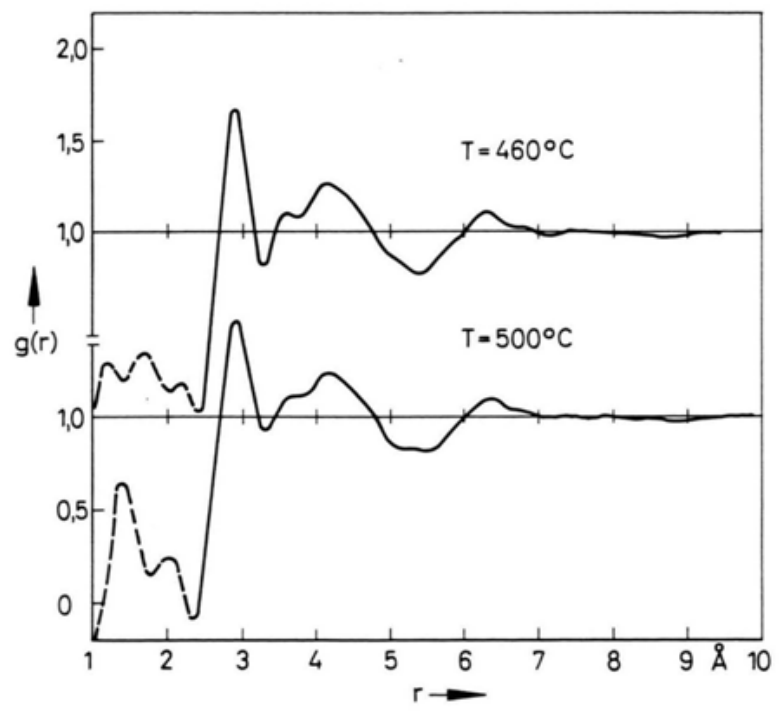

Fig. 4. Pair correlation functions calculated from the $\mathrm{X}$-ray scattering results shown in Fig. $2,0<Q<11.75 \AA^{-1}$.

Table 1. Position of first coordination shell $r^{1}$ and coordination number of nearest neighbours $N^{1}$.

\begin{tabular}{lcccc}
\hline & $T /{ }^{\circ} \mathrm{C}$ & $Q_{\max } / \AA^{-1}$ & $r^{1} / \AA$ & $N^{1}$ \\
\hline Neutron & 480 & 9.35 & 2.90 & 2.68 \\
diffraction & 550 & 9.35 & 2.90 & 2.64 \\
X-ray & 460 & 11.75 & 2.90 & 2.33 \\
diffraction & 500 & 11.75 & 2.90 & 2.44 \\
\hline
\end{tabular}

\section{Discussion}

\subsection{Previous Investigations}

A summary of characteristic results from previous structure investigations is given in Table 2 .

Nearly two decades ago Cabane and Friedel [6] proposed a local order model which was mainly based on neutron scattering experiments of Tourand and Breuil [7]. Between the melting point $\left(452^{\circ} \mathrm{C}\right)$ and approximately $600{ }^{\circ} \mathrm{C}$ they expected a gradual decomposition of the twofold chain structure of crystalline Te accompanied by a simultaneous growth of a network of threefold-coordinated sites having As-like local order.

The pair distribution functions from the early diffraction experiments with neutrons by Tourand and Breuil [7], Tourand [8] and with X-rays by Hoyer et al. [9] and Waseda et al. [10] are in all cases characterized by three well-resolved peaks at about $2.95 \AA$, 3.4-3.8 $\AA$ and 4.3-4.5 $\AA$. The corresponding coordination numbers are approximately 3,3 and $6-7$. No temperature dependence of the radius and the coordination number of the first coordination sphere has been observed in the temperature range above the melting point $[9,10]$. Moreover, a value of 2.85 for the first neighbour coordination number in supercooled liquid $\mathrm{Te}$ at $403{ }^{\circ} \mathrm{C}[8]$ suggested that the coordination never decreases appreciably below 3 . The meaning of the second peak around $3.4-3.8 \AA$ was disputed by Enderby and Gay [11]. By repeated numerical inversion and reinversion of their diffraction data it was shown that the structure factor $S(Q)$ can be regenerated from a pair correlation function $g(r)$ in which the only peaks resolved are at $3.01 \AA$ and at $4.52 \AA$.

Bellissent and Tourand [12] found a first coordination number 3 from neutron scattering experiments at $475^{\circ} \mathrm{C}$. The structure factor was modelled on the basis of the divalent lattice of crystalline Te. Nguyen et al. [13] determined a first coordination number 3.1. In both papers $[12,13]$ the pair correlation function exhibits a pronounced shoulder in the range of 3.4-3.8 $\AA$. The structure factor of liquid tellurium has been described by Bellissent [14] using a quasi-crystalline model on the basis of the three-fold-coordinated Asstructure.

From their neutron scattering experiments Takeda et al. [4] found a temperature dependent variation of the structure of liquid tellurium. At $460{ }^{\circ} \mathrm{C}$ the position of the first peak is $2.82 \AA$ and its area corresponds to 2.6 atoms. These quantities change gradually and 
Table 2. Summary of characteristic features from previous structure investigations.

\begin{tabular}{|c|c|c|c|c|c|c|}
\hline Author & $\mathrm{T} /{ }^{\circ} \mathrm{C}$ & $r^{1} / \AA$ & $N^{1}$ & $Q_{\max } / \AA^{-1}$ & $\begin{array}{l}\text { Experiment } \\
\mathrm{x}, \mathrm{n}\end{array}$ & $\begin{array}{l}\text { Shoulder } \\
\text { or peak }\end{array}$ \\
\hline Tourand and Breuil [7] & $\begin{array}{l}575 \\
775\end{array}$ & $\begin{array}{l}2.97 \\
3.02\end{array}$ & $\begin{array}{l}3.16 \\
3.37\end{array}$ & $\begin{array}{l}9.5 \\
9.5\end{array}$ & $\begin{array}{l}\mathrm{n} \\
\mathrm{n}\end{array}$ & $\begin{array}{l}\text { yes } \\
\text { yes }\end{array}$ \\
\hline Tourand [8] & $\begin{array}{l}403 \\
457\end{array}$ & $\begin{array}{l}2.89 \\
2.92\end{array}$ & $\begin{array}{l}2.85 \\
2.94\end{array}$ & $\begin{array}{l}9.5 \\
9.5\end{array}$ & $\begin{array}{l}\mathrm{n} \\
\mathrm{n}\end{array}$ & $\begin{array}{l}\text { yes } \\
\text { yes }\end{array}$ \\
\hline Hoyer et al. [9] & $\begin{array}{l}480 \\
620\end{array}$ & $\begin{array}{l}2.95 \\
2.96\end{array}$ & $\begin{array}{l}2.98 \\
3.01\end{array}$ & $\begin{array}{l}13.5 \\
13.5\end{array}$ & $\begin{array}{l}x \\
x\end{array}$ & $\begin{array}{l}\text { yes } \\
\text { yes }\end{array}$ \\
\hline Waseda and Tamaki [10] & $\begin{array}{l}470 \\
570\end{array}$ & $\begin{array}{l}2.95 \\
2.96\end{array}$ & $\begin{array}{l}3.03 \\
3.14\end{array}$ & - & $\begin{array}{l}\mathrm{x} \\
\mathrm{x}\end{array}$ & $\begin{array}{l}\text { yes } \\
\text { yes }\end{array}$ \\
\hline Enderby and Gay [11] & 500 & 3.01 & 3 & 12 & $\mathrm{n}$ & no \\
\hline Nguyen et al. [13] & 520 & - & 3.1 & $12^{*}$ & $\mathrm{n}$ & yes \\
\hline Takeda et al. [4] & $\begin{array}{l}460 \\
730\end{array}$ & $\begin{array}{l}2.82 \\
2.91\end{array}$ & $\begin{array}{l}2.6 \\
3.1\end{array}$ & $\begin{array}{l}9^{*} \\
9^{*}\end{array}$ & $\begin{array}{l}\mathrm{n} \\
\mathrm{n}\end{array}$ & $\begin{array}{l}\text { yes } \\
\text { yes }\end{array}$ \\
\hline Menelle et al. [15] & $\begin{array}{l}410 \\
800\end{array}$ & $\begin{array}{l}2.82 \\
2.85\end{array}$ & $\begin{array}{l}2.42 \\
3.06\end{array}$ & $\begin{array}{l}15 \\
15\end{array}$ & $\begin{array}{l}\mathrm{n} \\
\mathrm{n}\end{array}$ & $\begin{array}{l}\text { no } \\
\text { no }\end{array}$ \\
\hline \multirow[t]{2}{*}{ Menelle et al. [16] } & 330 & $2.8^{*}$ & $\begin{array}{l}2.1^{* *} \\
2.1^{* * *}\end{array}$ & 15 & $\mathrm{n}$ & no \\
\hline & 850 & $2.8^{*}$ & $\begin{array}{l}2.1 * * \\
2.7^{* * *}\end{array}$ & 15 & $\mathrm{n}$ & yes \\
\hline Inui et al. [21] & 460 & 2.83 & 2 & & EXAFS & yes \\
\hline
\end{tabular}

* Approximate value taken from a figure of the quoted reference. $-{ }^{* *}$ From integration up to $3.11 \AA .-{ }^{* * *}$ From integration up to the first minimum (see text).

are $2.91 \AA$ and 3.1 atoms at $730^{\circ} \mathrm{C}$. The melt was explained to be a mixture of non-metallic twofoldcoordinated regions, having a chain-like structure similar to liquid selenium, and of metallic threefoldcoordinated groupings.

By means of thermal neutron scattering with a high maximum momentum-transfer Menelle et al. [15] determined the short-range order in a temperature intervall covering $50 \mathrm{~K}$ of the supercooled region and about $300 \mathrm{~K}$ above the melting point. Within this range the radius of the first coordination shell is constant while the coordination number increases from 2.41 at $410^{\circ} \mathrm{C}$ to 3.06 at $800{ }^{\circ} \mathrm{C}$. The steepest rise takes place already in the supercooled liquid. Above approximately $500{ }^{\circ} \mathrm{C}$ the coordination number remains practically constant. From this behaviour a sharp semiconductor-metal transition, mainly occurring in the supercooled liquid, has been deduced [15]. In a more recent paper Menelle et al. [16] reported about neutron scattering experiments covering the wide temperature range from $330^{\circ} \mathrm{C}$ to $850^{\circ} \mathrm{C}$ which leads to a splitting of the first coordination shell, a low distance part and a higher distance part. While integration from 2.43 to $3.11 \AA$ leads to 2.1 nearest neighbours for all temperatures, the integration taken from $2.43 \AA$ to the first minimum shows an increase of the coordination number from 2.1 at the lowest temperature to 2.7 at $700{ }^{\circ} \mathrm{C}$.

Enderby and Barns [17] review the results of the more recent neutron diffraction experiments. Based on the findings from Hawker et al. [18], Menelle et al. [15] and of Welland et al. [19] they state that the oftenquoted work of Tourand and Breuil [7] is seriously in error and conclude that nothing in the structural data for liquid tellurium contradicts an atomic arrangement with a number of two or even less covalent bonds per atom on average.

Hafner [20,21] presented a molecular-dynamics calculation of the structure of molten Te. The analysis of the local topology of the model has shown that the atoms form a network of entangled broken chains. The first coordination number is about 2.5 just above the melting point [20]. In contrast to liquid As, where two different types of local environment exist, in liquid Te the bond-angle distribution function shows a single peak at the bond-angle of the crystalline Te-structure [21].

From EXAFS measurements Inui et al. [22] state that for liquid tellurium no single-shell model is good enough to fit the observed EXAFS spectrum, while the spectrum is better reproduced by describing the first neighbour shell as the superposition of two Gaussian 
curves centered at 2.83 and $3.03 \AA$. The total coordination number is estimated to be approximately 2 . A well-defined intermediate peak, located at $3.48 \AA$, has been found.

As can be seen from comparison of the quoted results there are at least three remaining questions:

(i) What is the coordination number of first neighbours, does it tend to be two or three?

(ii) Does the short-range order change with increasing temperature or not?

(iii) What is the nature of the intermediate peak or shoulder in the region between 3.4 and $3.8 \AA$ ?

\subsection{Dependence of the First Coordination Number and of the Shape of the Second Maximum of $g(r)$ on the Limits of the Fourier Transformation}

As is well known, the scattering pattern can be measured only over some finite range of $Q$, and consequently the resulting distribution functions differ from the ideal distribution functions.

The structure factors from our neutron and X-ray experiments have been measured down to $Q_{\min }=$ $1 \AA^{-1}$. For smaller $Q$-values a parabolic extrapolation

$$
S(Q)=S(0)+A Q^{2}
$$

has been employed, which, according to Evans and Sluckin [23], is a good approximation for $S(Q)$ at small momentum transfers for both, metallic and nonmetallic monatomic melts. In (1) $S(0)=0.062$ is the value given by Blairs and Joasoo [23] and $A$ was determined by fitting to the experimental data at $Q_{\min }$. It has been shown that a variation of $A$ and $S(0)$ is only of small influence on the pair correlation function while the extrapolation itself is not negligible [2]. In Fig. 5 two pair correlation functions are shown which were calculated from the $\mathrm{X}$-ray structure factor at $460^{\circ} \mathrm{C}$ using lower integration limits of $0 \AA^{-1}$ and $1 \AA^{-1}$. In both cases the upper integration limit was $11.75 \AA^{-1}$. The difference curve between the two functions, which is also shown in Fig. 5, illustrates the influence of termination at $Q_{\min }>0$. The region around the first coordination shell, especially its left flank, is rather strongly affected. Truncation at $Q_{\min }>0$ increases the correlation function in this $r$-region. While integration with $Q_{\min }=0 \AA^{-1}$ leads to $N^{1}=2.33$, the truncation at $Q_{\min }=1 \AA^{-1}$ causes a magnification of $N^{1}$ by about $20 \%$.

In Fig. 6 five pair correlation functions are shown which were calculated from the X-ray structure factor

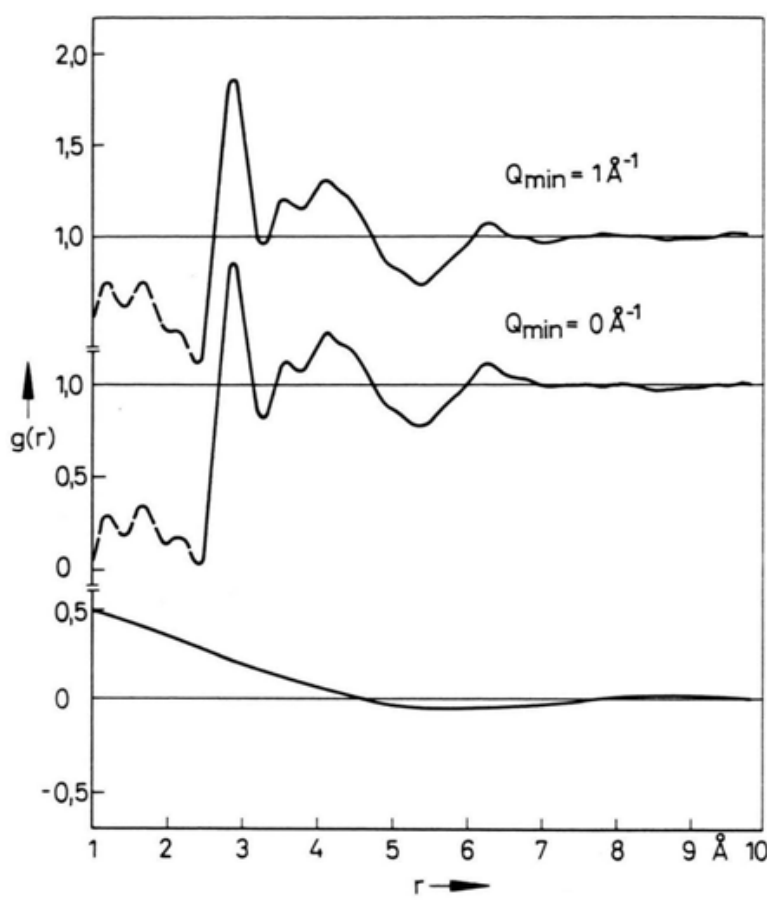

Fig. 5. Pair correlation functions calculated with different integration limits $Q_{\min }$. The difference between the two functions is shown at the bottom.

$\left(460{ }^{\circ} \mathrm{C}\right)$ in order to demonstrate the influence of $Q_{\max }$. The height of the first peak increases with increasing $Q_{\max }$ while the peak width decreases. The coordination number of first neighbours as a function of the integration limit of the Fourier transformation is given in Figure 9. A noticeable decrease with increasing $Q_{\max }$ is found.

The $r$-region around the second coordination sphere is affected by the truncation, as can clearly be seen from Figure 6. On both the left and right side of the second maximum ghost peaks are superimposed. Therefore a straightforward interpretation may be misleading.

One possible way to estimate truncation effect errors is to make an educated guess on $S(Q)$ for $Q>Q_{\max }$. For the extrapolation we used the function

$$
S(Q)=c_{1} \cos \left(c_{2} Q-c_{3}\right) \exp \left(-c_{4} Q\right) / Q+1,
$$

which enables us to obtain an analytical expression for the pair correlation function also for $Q_{\max }=\infty$. The parameters $c_{1} \ldots c_{4}$ have been determined by a least squares fit of the experimental $S(Q)$ in the range $6.65 \AA^{-1}<Q<12.0 \AA^{-1}$. In the case of the $\mathrm{X}$-ray 


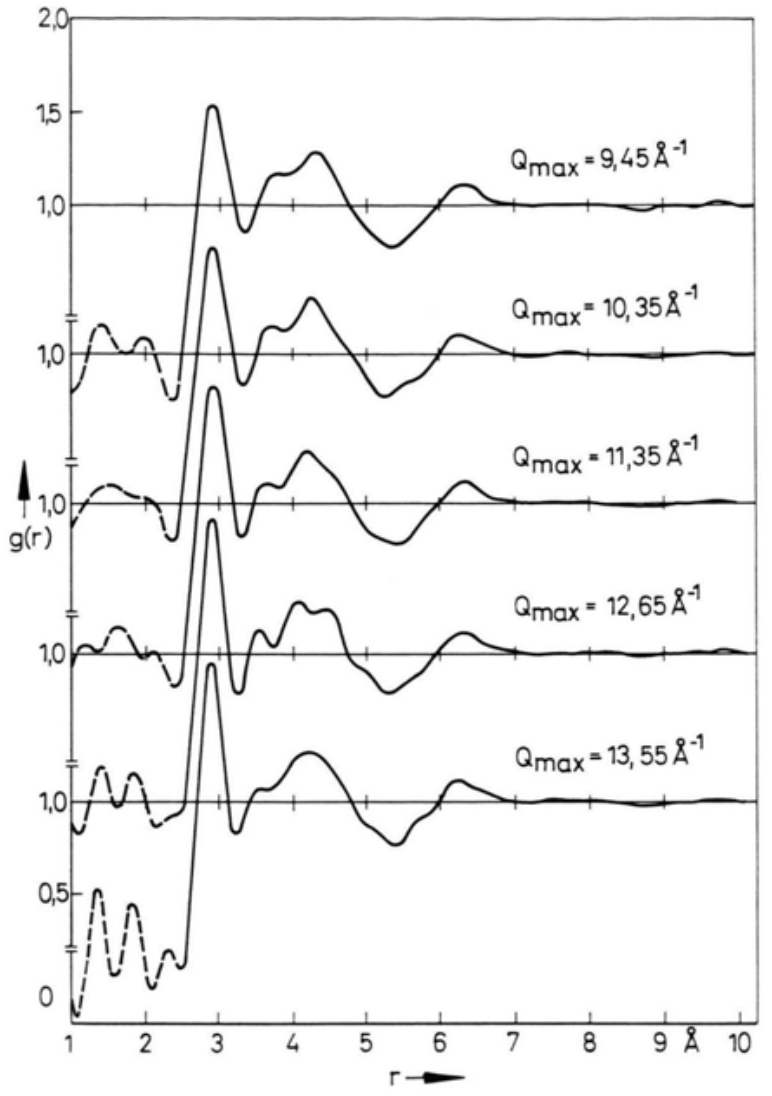

structure factor at $460{ }^{\circ} \mathrm{C}$ the parameters are

$$
\begin{array}{ll}
c_{1}=0.803 \AA^{-1}, & c_{3}=20.836, \\
c_{2}=2.921 \AA, & c_{4}=0.153 \AA .
\end{array}
$$

In Fig. 7 the experimental data and the fit according to (2) with the above parameters are shown. The functions $Q(S(Q)-1)$ are drawn. Pair correlation functions which were calculated from a set of $S(Q)$ data consisting of extrapolated values according to (1) for $0 \AA^{-1}<Q<1 \AA^{-1}$, experimental $\mathrm{X}$-ray scattering data at $460{ }^{\circ} \mathrm{C}$ for $1 \AA^{-1}<Q<6.65 \AA^{-1}$ and extrapolated values according to (2) in the range $6.65 \AA^{-1}<Q<Q_{\max }$ are shown in Figure 8. Up to $Q_{\max }=13.55 \AA^{-1}$ the same integration limits have been chosen as for the pair correlation functions given in Fig. 6, which were calculated directly from the experimental X-ray data. A comparison of pair correlation functions from Figs. 6 and 8 proves the reliability of the structure factor modelling with the help of (2).

The calculated coordination numbers give a additional support. They practically coincide with the coordination numbers which were determined from

Fig. 6. Pair correlation functions calculated from the X-ray structure factor at $460^{\circ} \mathrm{C}$ with different $Q_{\max }$.

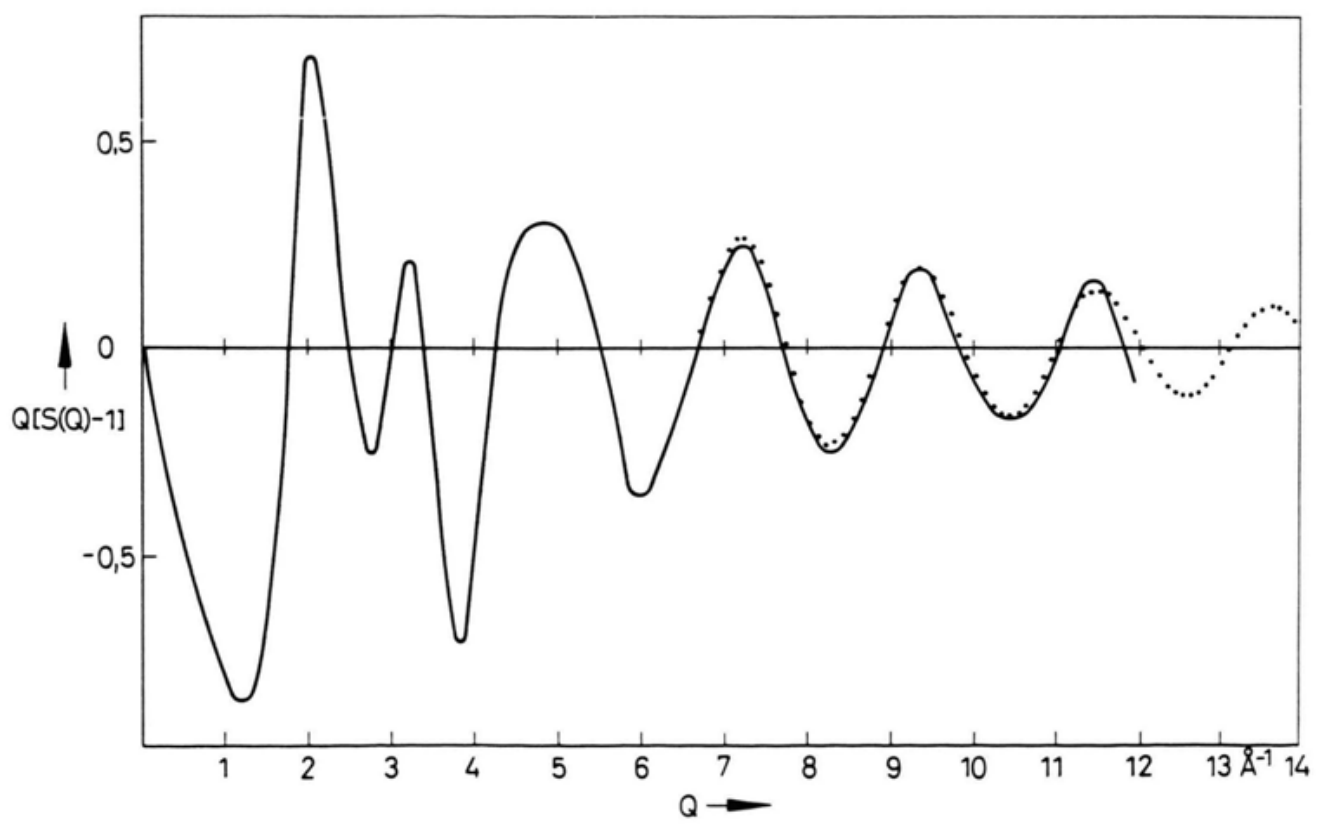

Fig. 7. X-ray diffraction data and fitted values, Eq. (2), - experimental data, $\cdots \cdots$ fitted values. 


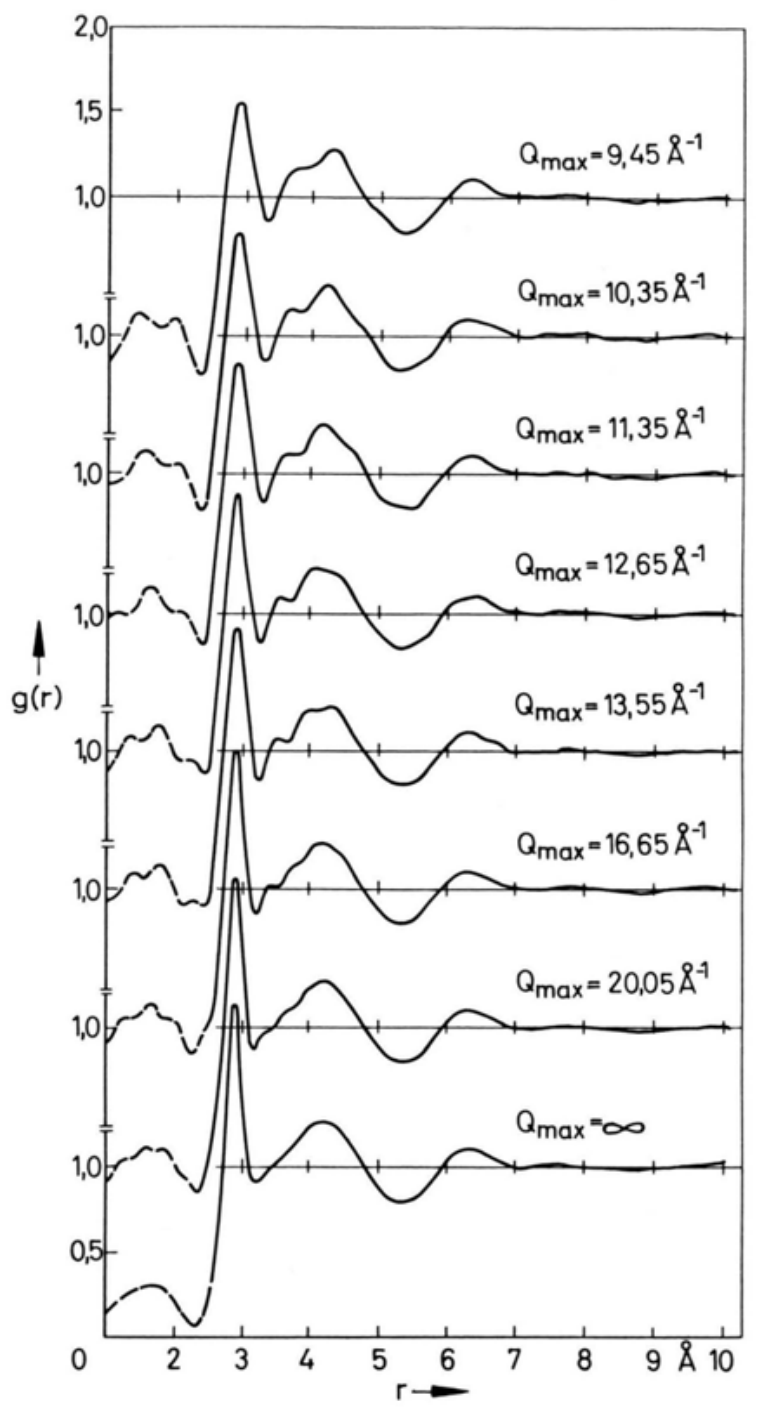

Fig. 8. Pair correlation functions calculated with fitted and extrapolated $S(Q)$ data.

the $4 \pi r^{2} g(r)$ functions of Figure 6. The comparison is shown in Figure 9. This behaviour for the $Q$-range $6.65 \AA^{-1}<Q<13.55 \AA^{-1}$, where the fitted $S(Q)$ produces practically the same correlation functions as the experimental one, gives some confidence to use the extrapolated $S(Q)$ data when investigating the truncation effect for $Q>13.55 \AA^{-1}$.

The truncations at $9.45 \AA^{-1}, 10.35 \AA^{-1}, 11.35 \AA^{-1}$, $12.65 \AA^{-1}, 13.55 \AA^{-1}, 16.55 \AA^{-1}, 20.05 \AA^{-1}$ and the integration up to infinity enlighten two points:

(i) The coordination number $N^{\mathrm{I}}$ continuously decreases with increasing $Q_{\max }$ up to $Q_{\max }=20.05 \AA^{-1}$. It

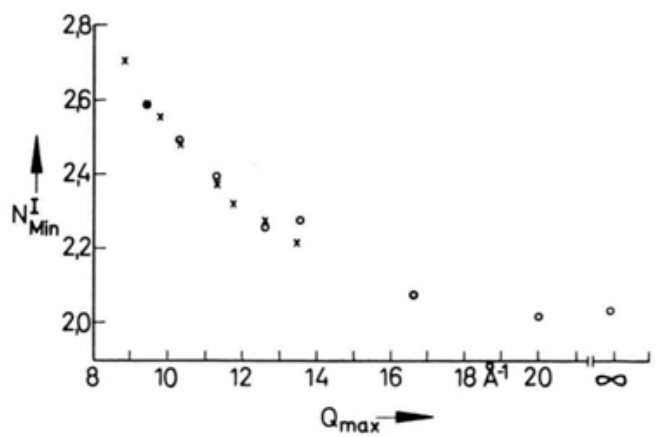

Fig. 9. Coordination number of the first shell as a function of $Q_{\max } \times 4 \pi r^{2} \varrho_{0} g(r)$ from experimental X-ray data, $\circ 4 \pi r^{2} \varrho_{0} g(\mathrm{r})$ from $S(Q)$ fitted and extrapolated with the help of (2) in the range $6.65 \AA^{-1}<Q<Q_{\max }$.

reaches the value 2, which is characteristic for the short-range order of solid Te (cf. Figure 9). The steepest increase is observed for integration limits lower than approximately $12 \AA^{-1}$. This behaviour is in accordance with the general predictions of Hosemann et al. [23].

(ii) The shape of the second maximum of $g(r)$ is clearly influenced by the upper integration limit even up to high maximum momentum transfers. This means in particular: the deep splitting of the second maximum, which occurs for lower $Q_{\max }$, is obviously caused by the truncation effect. As can be seen from the $g(r)$ function, which has been calculated by integration up to infinity, the splitting has nearly disappeared. As a residual structure a weakly pronounced shoulder remains. Of course this shoulder or at least asymmetry indicates that between the two main maxima, located at $2.90 \AA^{-1}$ and about $4.25 \AA$, respectively, a further coordination sphere around about $3.5 \AA$ is to be expected.

\section{Conclusion}

Truncation of the Fourier transformation at $Q_{\min }>0$ leads to increased values of the coordination number of nearest neighbours. While there is no detectable influence of the integration limit $Q_{\max }$ on the radius of the first coordination shell, it becames clear that the area under the first peak of the radial distribution function is affected. A strong influence has been found for integration limits lower than approximately $12 \AA^{-1}$. A smaller but noticeable influence is present even up to $Q_{\max }$-values of about $20 \AA^{-1}$. From the observed 
dependence of $N^{1}$ on $Q_{\max }$ one can estimate that the real bond number of liquid tellurium is two. This value of the coordination number is reached by integration of the extrapolated structure factor up to infinity.

The first and second coordination shell are not well separated, and consequently the determination of the first coordination number can not be done unambiguously. Hence the values of "coordination numbers" determined from radial distribution functions of liquid tellurium have to be handled with care.

For both experiments, neutron and X-ray scattering, the observed radius of the first coordination shell

[1] R. Bellissent, J. Non-Cryst. Solids 97-98, 1263 (1987).

[2] H. Neumann, W. Matz, and W. Hoyer, Experimentelle Technik der Physik 36, 105 (1988).

[3] H. Neumann et al., phys. stat. solidi 90 a, 489 (1985).

[4] Sh. Takeda, Sh. Tamaki, and Y. Waseda, J. Phys. Soc. Japan 53, 3830 (1984).

[5] H. Thurn and J. Ruska, J. Non-Cryst. Solids 22, 331 (1976).

[6] B. Cabane and J. Friedel, J. Phys. Paris 32, 73 (1971).

[7] G. Tourand and M. Breuil, J. Phys. Paris 32, 813 (1971).

[8] G. Tourand, Phys. Letters 54, 209 (1975).

[9] W. Hoyer, E. Thomas, and M. Wobst, Z. Naturforsch. 30 a, 235 (1975).

[10] Y. Waseda and S. Tamaki, Z. Naturforsch. 30a, 1655 (1975).

[11] J. E. Enderby and M. Gay, J. Non-Cryst. Solids 35-36, 1269 (1980).

[12] R. Bellissent and G. Tourand, J. Non-Cryst. Solids 35-36, 1221 (1980).

[13] V. T. Nguyen, M. Gay, J. E. Enderby, R. J. Newport, and R. A. Howe, J. Phys. C: Solid State Phys. 15, 4627 (1982).

[14] R. Bellissent, Thesis, L'Universite Pierre et Marie Curie, Paris 1981. and its coordination number show no dependence on temperature in the investigated range 480 to $550^{\circ} \mathrm{C}$. None of our experiments contradicts the picture of a chain-like structure with two covalent bonds per atom. However, it seems to us that the only straightforward way to resolve the controversy over the existence of twofold or threefold coordination of liquid tellurium is from a neutron scattering experiment covering a very wide momentum transfer range, with $Q_{\max }$ larger than at least $20 \AA^{-1}$.

[15] A.Menelle, R. Bellissent, and A. M. Flank, Europhys. Lett. 4, 705 (1987).

[16] A. Menelle, R. Bellissent, and A. M. Flank, Physica B 156-157, 174 (1989).

[17] J. E. Enderby and A. C. Barnes, Repts on Progress in Physics 53, 85 (1990).

[18] I. Hawker, R. A. Howe, and J. Enderby, Int. Conf. on the Electronic and Magnetic Properties of Liquid Metals (J. Keller, ed.), Mexico 1978, p. 262 (Mexico City: UNAM).

[19] M. E. Welland, M. Gay, and J. E. Enderby, Physics of Disordered Systems (D. Adler et al., eds.), Plenum Press, New York 1985 , p. 695

[20] J. Hafner, J. Phys., Cond. Matter 2, 903 (1990).

[21] J. Hafner, J. Non-Cryst. Solids 117-118, 18 (1990).

[22] M. Inui, K. Tamura, M. Yao, H. Endo, S. Hosokawa, and H. Hoshino, J. Non-Cryst. Solids 117-118, 112 (1990).

[23] R. Evans and T. J. Sluckin, J. Phys. C 14, 3137 (1981)

[24] S. Blairs and U. Joasoo, J. Inorg. Nucl. Chem. 42, 1555 (1980).

[25] R. Hosemann, K. Lemm, and H. Krebs, Z. Phys. Chem. N.F. 41, 121 (1964). 